\title{
Desarrollo y validación de un instrumento para medir intimidad emocional en relaciones de pareja
}

\author{
Juan Aníbal González-Rivera \\ Psy.D. en Psicología Clínica \\ Ponce Health Sciences University, San Juan University Center \\ Puerto Rico \\ Correo electrónico: dr.juananibalgonzalez@outlook.com
}

Recibido: 24/09/2018

Evaluado: 24/01/2019

Aceptado: 01/04/2019

\section{Resumen}

El presente estudio analiza las propiedades psicométricas de la Escala de intimidad emocional en relaciones de pareja en una muestra de adultos puertorriqueños. Un total de 382 puertorriqueños participaron en este estudio de carácter exploratorio y psicométrico. Los resultados confirmaron que la escala posee una estructura de dos factores. Estos factores fueron identificados como cercanía emocional y distancia emocional. Los 10 ítems de la escala cumplieron con los criterios de discriminación y cargas factoriales apropiadas (cinco ítems por factor). El índice de confiabilidad alfa de Cronbach de la escala fue .90. Estos resultados sugieren que el instrumento tiene el potencial para medir este constructo en adultos puertorriqueños. Además, la escala permitirá el avance de nuevas investigaciones sobre intimidad emocional en parejas puertorriqueñas y latinoamericanas.

\section{Palabras clave}

intimidad emocional, parejas, confiabilidad, propiedades psicométricas, validación

1 Para citar este artículo: González-Rivera, J.A. (2020). Desarrollo y validación de un instrumento para medir intimidad emocional en relaciones de pareja. Informes Psicológicos, 20(2), pp. 11-22 http://dx.doi.org/10.18566/infpsic. v20n2a1 


\section{Development and validation of an instrument to measure emotional intimacy in couple relationships}

\section{Abstract}

This study analyzes the psychometric properties of the Emotional Intimacy Scale (EIS) in couple relationships in a sample of Puerto Rican adults. A total of 382 Puerto Ricans participated in this exploratory and psychometric study. The results confirmed that the scale has a two-factor structure. These factors were identified as emotional closeness and emotional distance. The 10 items on the scale met the criteria for discrimination and appropriate factor loads (five items per factor). The Cronbach alpha reliability index of the scale was .90. These results suggest that the instrument has the potential to measure this construct in Puerto Rican adults. In addition, the scale will allow the advancement of new research on emotional intimacy in Puerto Rican and Latin American couples.

Keywords emotional intimacy, couples, reliability, psychometric properties, validation.

\section{Desenvolvimento e validação de um instrumento para medir a intimidade emocional nos relacionamentos do casal}

\section{Resumo}

0 presente estudo analisa as propriedades psicométricas da Escala de intimidade emocional nas relações de casal em uma amostra de adultos porto-riquenhos. Um total de 382 porto-riquenhos participou deste estudo exploratório e psicométrico. Os resultados confirmaram que a escala possui uma estrutura de dois fatores. Esses fatores foram identificados como proximidade emocional e distância emocional. Os 10 itens da escala atenderam aos critérios de discriminação e cargas fatoriais apropriadas (cinco itens por fator). 0 índice de confiabilidade alfa de Cronbach da escala foi de 0,90. Esses resultados sugerem que 0 instrumento tem o potencial de medir esse construto em adultos porto-riquenhos. Além disso, a escala permitirá 0 avanço de novas pesquisas sobre intimidade emocional em casais porto-riquenhos e latino americanos.

Palavras chave intimidade emocional, casais, confiabilidade, propriedades psicométricas, validação. 


\section{ntroducción}

El estudio empírico de variables asociadas al conflicto en las relaciones de pareja es limitado debido a la carencia de instrumentos fiables y válidos que examinen las mismas (loseba \& MartinezPampliega, 2009). La mayoría de los instrumentos que existen se limitan al constructo satisfacción o ajuste en la relación de pareja, dejando al descubierto la necesidad de instrumentos que examinen otros elementos importantes asociados al conflicto o la separación en las relaciones de pareja. Al respecto, DeRogatis (2008) recomienda desarrollar instrumentos breves de fácil administración y con adecuados índices de validez y consistencia interna. El desarrollo de instrumentos de esta índole, y con estas características, facilitaría la tarea investigativa de temas asociados al conflicto en las relaciones de pareja como, por ejemplo, la falta de intimidad emocional.

Tradicionalmente, la intimidad -desde un sentido amplio del término- suele interpretarse desde una perspectiva bidimensional: aspecto sexual y emocional (Laurenceau, Barrett, \& Pietromonaco, 1998). Mientras que la intimidad sexual es aquella asociada con el afecto en general, el contacto, la cercanía física y la actividad sexual (Dandurand \& Lafontaine, 2013), la emocional está atada a un sentimiento de cercanía con otra persona que lleva a una tendencia a auto-revelarse al otro (Schaefer \& Olson, 1981). Sin embargo, la intimidad emocional se identifica como una característica propia de la relación de pareja, y la sexual es más una característica del individuo en la relación (Vohs \& Baumeister, 2004).

De acuerdo a la teoría triangular del amor de Sternberg (1988), la intimidad (aspecto emocional del amor) son aquellos sentimientos dentro de una relación que promueven el acercamiento, el vínculo y la conexión, tales como el afecto, revelaciones mutuas, secretos compartidos únicamente por la pareja, entre otros. Según la teoría del autor, la intimidad se desarrolla cuando se experimentan una cantidad considerable de estos sentimientos, siendo esta cantidad seguramente vertible según la persona y el contexto. Por su parte, Schaefer y Olson (1981) describen cinco tipos de intimidad: (1) emocional, la cual se refiere a experimentar sentimientos de cercanía; (2) social, asociada a la experiencia de tener amigos y actividades sociales comunes; (3) intelectual, coligada a la experiencia de compartir ideas; (4) sexual; y (5) recreacional, la cual representa el compartir experiencias recreativas.

El principal aspecto sobre la intimidad emocional que todas las definiciones y acercamientos teóricos parecen enfatizar es la sensación de cercanía psicológica que se desarrolla a través de la comunicación entre dos personas. Si esta cercanía emocional no se genera o se deteriora, podría provocar una sensación de distanciamiento emocional -sentimiento de alejamiento y desapego entre la pareja (Beckerman, 2002). En este sentido, un alto nivel de intimidad emocional se reflejará mediante un elevado grado de confianza entre los miembros de una relación, conductas amorosas e interacciones gratificantes (Hand, Thomas, Buboltz, Deemer, \& Buyanjargal, 2013). Investigaciones han demostrado que la intimidad emocional se asocia fuerte y significativamente con 
la satisfacción en la relación de pareja (Dandeneau \& Johnson, 1994; Dandurand \& Lafontaine, 2013; Greeff \& Malherbe, 2001). De hecho, algunos investigadores han encontrado que la intimidad emocional aporta más al bienestar de la pareja que la intimidad física o sexual (Dandurand \& Lafontaine, 2013; ZimmerGembeck, Arnhold, \& Connolly, 2014).

Desafortunadamente, la cantidad de instrumentos que existen en español para medir el constructo intimidad emocional es limitada (la mayoría son en inglés). En castellano, hasta donde es de nuestro conocimiento, contamos con tres instrumentos para medir intimidad emocional. Aunque debemos señalar que en los tres casos se mide el constructo en una subescala del instrumento general. En primer lugar, la Escala de Compromiso, Intimidad, Romance y Amor (ECIRA) desarrollada por Maureira (2016) para medir cuatro componentes del amor de pareja (compromiso, intimidad, romance y amor). La ECIRA fue validada en una muestra de 124 jóvenes chilenos y está constituida por 28 reactivos, correspondiendo siete preguntas a cada componente.

Por su parte, la Escala Triangular del Amor fue desarrollada originalmente en inglés por Sternberg (1986) y validada en español por Mazadiego y Garcés (2011) en una muestra de mil estudiantes universitarios veracruzanos. El instrumento constó de 45 reactivos, 15 para cada uno de los tres componentes (intimidad, pasión y compromiso). Por último, se encuentra la versión en español del Personal Assessment of Intimacy in Relationships (PAIR). Este inventario, desarrollado por Schaefer y Olson (1981), evalúa la intimidad conyugal y el ajuste de la pareja. Consta de 36 reactivos que evalúan cinco aspectos de la intimidad conyugal: social, emocional, sexual, intelectual y recreacional. Una de las bondades del PAIR es que mide percepción (sensación actual) y expectativas (sensación buscada o ideal) en relación a los cinco aspectos de la intimidad.

Para contextos anglosajones existen varias escalas e inventarios que miden este constructo y otros relacionados. Entre ellos se encuentran el Quality of Relationships Inventory (Pierce, 1994), el PAIR (versión en inglés), el Intimate Relationship Scale (Hetherington \& Soeken, 1990), y el Waring Intimacy Questionnaire (Waring \& Reddon, 1983). Tanto las medidas que existen en inglés como las tres disponibles en español, tienen la particularidad de ser instrumentos bastante extensos lo cual dificultad su utilización en ciertos contextos.

Teniendo en consideración la carencia de instrumentos breves en español para medir este constructo, el presente trabajo tuvo como objetivo principal desarrollar y validar un instrumento que mida intimidad emocional desde un marco referencial de dos factores: cercanía emocional y distancia emocional. A estos efectos se diseñó un estudio para evaluar las propiedades psicométricas del instrumento y comprobar si el mismo mantiene la estructura bifactorial utilizada para su desarrollo. Esta investigación cuenta con tres objetivos principales: (1) analizar la construcción lógica del instrumento mediante análisis factorial exploratorio, (2) examinar la capacidad de discriminación de los ítems; (3) evaluar la confiabilidad del instrumento, y (4) explorar la validez convergente del instrumento a través de la asociación con la variable satisfacción en la relación de pareja. 


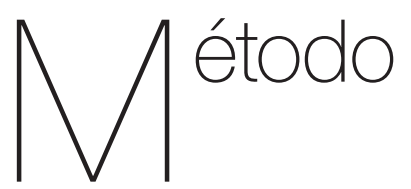

\section{Diseño}

Para lograr el objetivo de este trabajo investigativo, se utilizó un diseño no experimental, transversal, de tipo instrumental (Ato, López, \& Benavente, 2013; Montero \& León, 2007). Este diseño se utiliza en aquellas investigaciones que analizan las propiedades psicométricas de instrumentos de medida psicológicos.

\section{Participantes}

Se reclutó una muestra no probabilística de 382 adultos puertorriqueños, seleccionados por disponibilidad. La edad promedio de los participantes fue de 35.72 (DE $=10.59$ ) y los años promedio de convivencia con su pareja fue de 9.59 (DE = 9.96). En la Tabla 1 se presentan los datos sociodemográficos de la muestra.

Tabla 1.

Información sociodemográfica de los participantes

\begin{tabular}{lcc}
\hline Variables & $f$ & $\%$ \\
\hline Género & & \\
$\quad$ Femenino & 275 & 72.0 \\
$\quad$ Masculino & 107 & 28.0 \\
Ingresos anuales & & \\
25,000 o menos & 232 & 60.7 \\
$26,000-50,000$ & 107 & 28.0 \\
51,000 - 100,000 & 27 & 7.1 \\
101,000 0 más & 16 & 4.2 \\
Tipo de Relación & & \\
Matrimonio & 205 & 53.7 \\
$\quad$ Convivencia (Unión libre) & 177 & 46.3 \\
\hline
\end{tabular}

\begin{tabular}{lcc} 
Continuación & & \\
\hline Variables & $f$ & $\%$ \\
\hline Preparación Académica & & \\
$\quad$ Escuela Superior o menos & 36 & 9.5 \\
Asociado/curso técnico & 98 & 15.7 \\
Bachillerato & 151 & 39.5 \\
Maestría & 73 & 19.1 \\
Doctorado & 24 & 6.3 \\
\hline
\end{tabular}

Nota: $n=382$.

\section{Instrumento}

\section{Cuestionario de Datos \\ Generales.}

Para identificar las características sociodemográficas de la muestra, se desarrolló un cuestionario de datos generales, que recogía información importante en cuanto a la edad, sexo, ingresos anuales, tipo de relación y preparación académica.

\section{Escala de Intimidad Emocional en Relaciones de Pareja.}

Este instrumento fue desarrollado por el autor para medir intimidad emocional desde un modelo de dos factores: cercanía emocional y distancia emocional. El mismo está constituido por 10 ítems con una escala de respuesta de cuatro puntos, cuyos límites lo constituyen las respuestas: Totalmente en desacuerdo (1) a Totalmente de acuerdo (4) (e.g., Generalmente, mi pareja comprende mis preocupaciones y situaciones personales; Me gustaría que mi pareja me demostrara mayor interés y cercanía emocional). Los ítems del factor distancia emocional tienen codificación inversa (de 4 a 1). El puntaje más bajo que se puede obtener es 10 y el más alto es 40, donde elevadas 
puntaciones sugieren una alta intimidad emocional.

\section{Escala de Evaluación de la} Relación de Pareja (Hendrick, 1988).

Este instrumento mide de forma global los sentimientos, pensamientos o conductas en la relación de pareja (e.g., ¿Qué tanto tu pareja satisface tus necesidades?; En general, ¿qué tan satisfecho/a estás con tu relación?). Consta de siete ítems con una escala de respuesta de cinco puntos que fluctúan de Nada a Totalmente. El rango posible es de 7 a 35 puntos. A mayor puntaje, mayor satisfacción y calidad en la relación de pareja exhibe la persona $(\alpha=.91)$.

\section{Procedimiento}

La fase de reclutamiento se llevó a cabo de forma electrónica y presencial desde octubre 2017 hasta mayo 2018. El muestreo presencial estuvo a cargo de estudiantes graduados de psicología, quienes entrevistaron un total de 97 participantes reclutados por disponibilidad. El reclutamiento electrónico comenzó con un anuncio pagado en Facebook. Este anuncio redirigía a los participantes a una encuesta en línea disponible en la plataforma PsychData. El 75\% ( $n=285)$ de la muestra se obtuvo mediante este proceso.

Una vez recopilados los datos, se examinaron las propiedades psicométricas del instrumento. Para esto, se utilizó el sistema estadístico IBM SPSS versión 24.0 (IBM Corporation, 2016) y el programa estadístico STATA versión 14.1 (StataCorp.,
2017). En IBM SPSS se realizaron análisis de reactivos para conocer su índice de discriminación, análisis de confiabilidad, análisis factorial exploratorio, correlaciones entre las puntuaciones totales de los factores identificados y correlaciones entre las escalas de intimidad emocional y evaluación de la relación de pareja para examinar la validez convergente del instrumento. En STATA se realizó el análisis factorial confirmatorio con el método de estimación de máxima verosimilitud y las correcciones de Satorra y Bentler (2001).

Para evaluar el ajuste del modelo del análisis factorial confirmatorio se utilizaron los siguientes índices de bondad de ajuste: chi-cuadrado $\left(\chi^{2}\right)$, error medio cuadrático de aproximación (RMSEA), Índice Tucker-Lewis (TLI) y el índice de ajuste comparativo (CFI). Para que exista un ajuste aceptable del modelo los valores de CFI y TLI deben ser $\geq .90$ y los valores del RMSEA deben ser $\leq .08$ (Byrne, 2010; Hu \& Bentler, 1999). Por su parte, los coeficientes de regresión de cada ítem en su respectivo factor debían superar el .50 (Hair, Black, Babin, Anderson, \& Tatham, 2006).

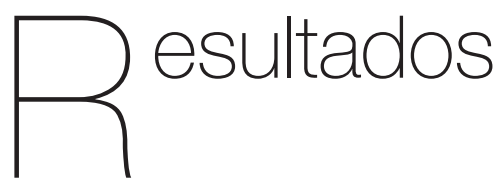

En primer lugar, se llevó a cabo un análisis factorial exploratorio utilizando el método de extracción de máxima verosimilitud con rotación oblicua para factores correlacionados, identificando aquellos factores que expliquen 5\% o más de la 
varianza como sugiere Hatcher (1994). Los resultados mostraron una estructura de dos factores que explicaban el $72 \%$ de la varianza de los datos originales, del cual el 54\% lo explica el factor 1 y el 18\% el factor 2. Las pruebas de Kaiser-MeyerOlkin $(\mathrm{KMO}=.912)$ y la prueba de esfericidad Bartlett $\left(X^{2}(45)=2368.019, \mathrm{p}<.000\right)$ apoyaron la adecuacidad de los datos de muestreo para el análisis. La Tabla 2 presenta las cargas factoriales obtenidas por los 10 ítems, cuya distribución por dimensión fue la siguiente: 5 ítems en el primer factor (cercanía emocional) y 5 ítems en el segundo factor (distancia emocional).
Luego, se realizaron dos análisis de discriminación a través del índice de correlación ítem-total (rbis): uno para calcular el índice del ítem al compararlo con la escala total y otro para calcular el índice del ítem en su respectivo factor. Además, se calculó la varianza explicada de los factores y escala total en los ítems. La Tabla 2 presenta los índices de discriminación del instrumento. Por último, se analizó la confiabilidad del instrumento mediante el índice Alfa de Cronbach y se examinó la relación entre los factores mediante la correlación $r$ de Pearson (ver Tabla 3).

Tabla 2

Índices de discriminación, varianza explicada y cargas factoriales.

\begin{tabular}{|c|c|c|c|c|c|c|c|c|}
\hline \multirow[t]{2}{*}{ Ítems } & \multicolumn{2}{|c|}{ Escala Total } & \multicolumn{3}{|c|}{ Factor 1: Cercanía } & \multicolumn{3}{|c|}{ Factor 2: Distancia } \\
\hline & $r_{\text {bis }}$ & $R^{2}$ & $r_{\text {bis }}$ & $R^{2}$ & Carga & $r_{\text {bis }}$ & $R^{2}$ & Carga \\
\hline Ítem 1 & .69 & .64 & .77 & .63 & .81 & - & - & - \\
\hline Ítem 2 & .69 & .68 & .82 & .68 & .89 & - & - & - \\
\hline Ítem 3 & .71 & .67 & .80 & .66 & .83 & - & - & - \\
\hline Ítem 4 & .58 & .50 & .70 & .50 & .76 & - & - & - \\
\hline Ítem 5 & .63 & .56 & - & - & - & .74 & .55 & .81 \\
\hline Ítem 6 & .64 & .59 & - & - & - & .75 & .59 & .83 \\
\hline Ítem 7 & .60 & .50 & .69 & .49 & .71 & - & - & - \\
\hline Ítem 8 & .60 & .54 & - & - & - & .72 & .54 & .82 \\
\hline Ítem 9 & .66 & .54 & - & - & - & .70 & .53 & .68 \\
\hline Ítem 10 & .77 & .66 & - & - & - & .78 & .62 & .72 \\
\hline
\end{tabular}

Nota: $r_{b i s}=$ Índice de discriminación; $R^{2}=$ Varianza explicada; Carga = carga factorial.

Tabla 3

Medias, desviaciones estándar, alfas y correlaciones ( $n=382)$.

\begin{tabular}{lcccccccc}
\hline & $M$ & $D E$ & $\alpha$ & $\alpha_{\text {est }}$ & 1 & 2 & 3 & 4 \\
\hline 1. Cercanía emocional & 14.53 & 4.34 & .90 & .90 & - & .51 & .86 & .63 \\
2. Distancia emocional & 11.69 & 4.71 & .89 & .89 & & - & .88 & .57 \\
3. Escala Total & 26.22 & 7.85 & .90 & .89 & & - & .69 \\
4. Calidad en la Relación & 25.59 & 5.73 & .90 & .91 & & & - \\
\hline
\end{tabular}

Nota. $M=$ media; $D E=$ desviación estándar; $\alpha=$ alfa de cronbach; $\alpha_{\text {est }}=$ alfa de Cronbach estandarizado. Todas las correlaciones fueron significativas a $p<.001$. 
Los 10 ítems de la escala fueron sometidos a un análisis factorial confirmatorio con ecuaciones estructurales. El modelo a prueba estaba conformado por dos factores latentes: cercanía emocional y distancia emocional (ver Figura 1). Los resultados arrojaron un ajuste adecuado para el modelo con las correspondientes liberaciones entre errores, $\chi^{2}=89.49$
(190) $\mathrm{p}<.001, \mathrm{RMSEA}=.07, \mathrm{CFI}=.98$, TLI $=.97, \chi_{\mathrm{sb}}^{2}=75.69$ (190) $p<.001, \mathrm{RM}-$ $\mathrm{SEA}_{\mathrm{sb}}=.06, \mathrm{CFI}_{\mathrm{sb}}=.98, \mathrm{TLIsb}=.97 . \mathrm{A}$ su vez, se examinaron los coeficientes de regresión de cada ítem, esperando que cada uno de estos fuese mayor a .50. Los coeficientes de regresión fluctuaron entre .73 y .87 (ver Tabla 4).

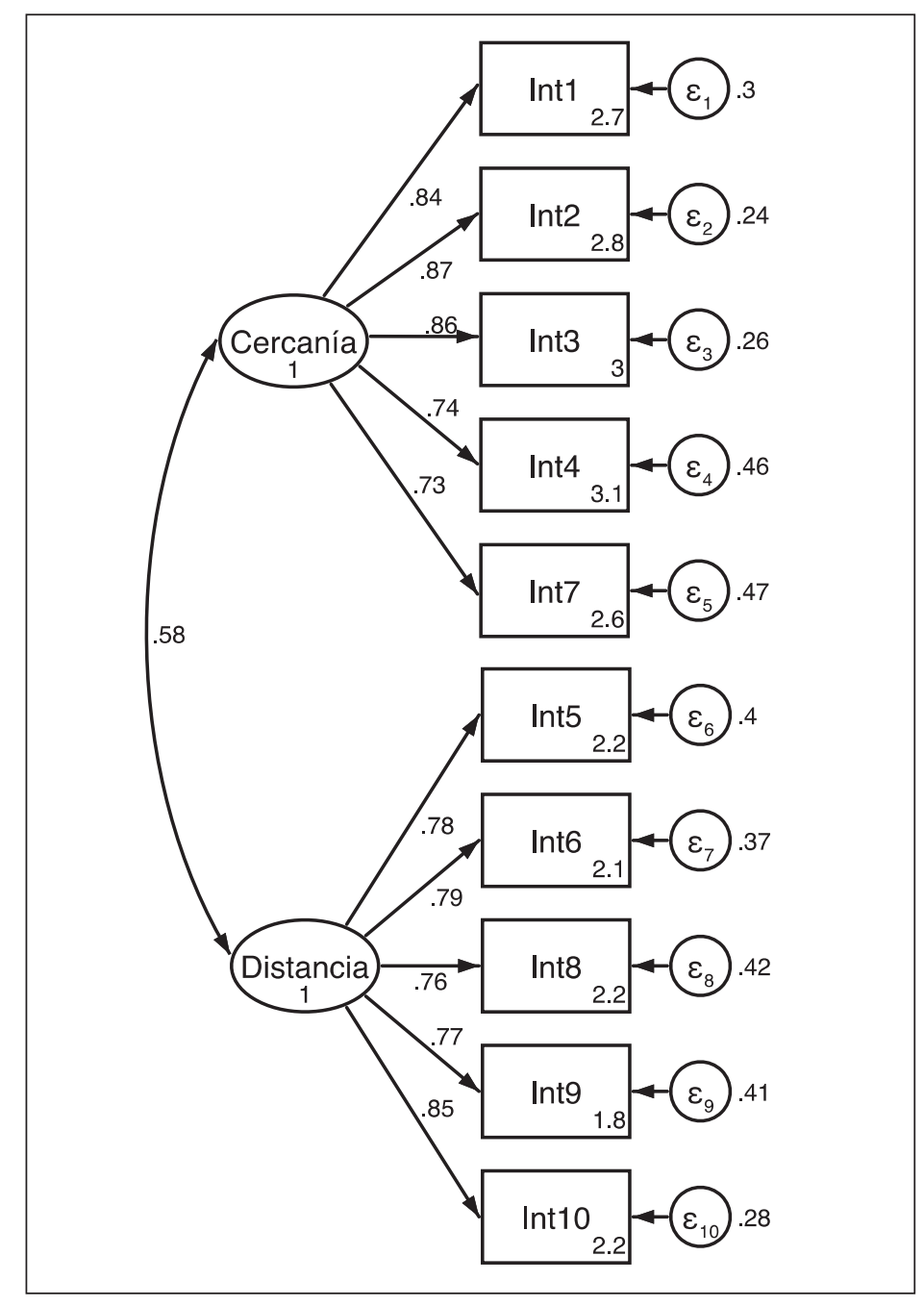

Figura 1. Modelo de dos factores de la Escala de Intimidad Emocional.

Por último, se examinó el grado de asociación entre la escala de intimidad emocional y la escala de evaluación de la relación de pareja para examinar la validez convergente del instrumento a través del coeficiente de correlación de Pearson. Se esperaba que el índice de correlación de la puntuación en la escala 
de intimidad emocional y sus subescalas correlacionasen moderadamente alta ( $>$ .50) y estadísticamente significativa con la puntuación en la escala de evaluación de la relación de pareja. Los datos obtenidos evidenciaron que los valores del coeficiente de correlación $r$ de Pearson entre las escalas fluctuaron entre .57 y .69 (ver Tabla 4). Esto confirma que la asociación entre las variables estudiadas se encuentra dentro de lo esperado y convergen entre sí.

Tabla 4

Coeficientes de regresión del análisis factorial confirmatorio y sus intervalos de confianza

\begin{tabular}{|c|c|c|}
\hline Ítems & $\beta$ & I.C. $95 \%$ \\
\hline $\begin{array}{l}\text { 1. Mi pareja me escucha atentamente cuando necesito } \\
\text { alguien con quien conversar o desahogarme. }\end{array}$ & .84 & {$[.78-.88]$} \\
\hline 2. Puedo expresarme tal y como soy sin sentirme juzgada/o por mi pareja. & .87 & {$[.83-.91]$} \\
\hline Ítems & $\beta$ & I.C. $95 \%$ \\
\hline $\begin{array}{l}\text { 3. Generalmente, mi pareja comprende mis } \\
\text { preocupaciones y situaciones personales. }\end{array}$ & .86 & {$[.82-.90]$} \\
\hline $\begin{array}{l}\text { 4. Siento la confianza suficiente como para contarle } \\
\text { a mi pareja cualquier cosa que me sucede. }\end{array}$ & .74 & {$[.67-.80]$} \\
\hline $\begin{array}{l}\text { 5. En ciertas ocasiones me siento descuidada/o } \\
\text { emocionalmente por mi pareja. }\end{array}$ & .78 & {$[.72-.84]$} \\
\hline $\begin{array}{l}\text { 6. Reconozco que en ocasiones me siento sola/o, } \\
\text { aun estando acompañada/o por mi pareja. }\end{array}$ & .79 & {$[.74-.85]$} \\
\hline $\begin{array}{l}\text { 7. Me siento en la confianza de expresarle a mi pareja mis } \\
\text { emociones y sentimientos sin que se torne a la defensiva. }\end{array}$ & .73 & {$[.66-.80]$} \\
\hline 8. En ciertas ocasiones me siento distante emocionalmente de mi pareja. & .76 & {$[.70-.82]$} \\
\hline $\begin{array}{l}\text { 9. Me gustaría que mi pareja me demostrara } \\
\text { mayor interés y cercanía emocional. }\end{array}$ & .77 & {$[.71-.83]$} \\
\hline $\begin{array}{l}\text { 10. En ciertos momentos percibo que no tengo } \\
\text { suficiente conexión emocional con mi pareja. }\end{array}$ & .85 & {$[.81-.89]$} \\
\hline
\end{tabular}

Nota: $\beta=$ coeficientes de regresión en su respectiva subescala; I.C. 95\% = intervalos de confianza de los coeficientes de regresión.

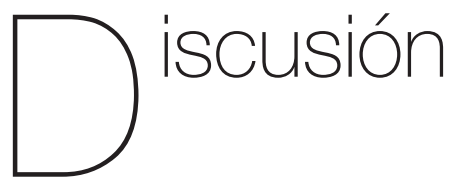

A partir de los resultados obtenidos, se puede establecer que el instrumento posee adecuadas características psicométricas para evaluar la intimidad emocional en relaciones de pareja desde un modelo bifactorial que mide cercanía emocional y distancia emocional. Estos dos factores miden de forma confiable un elemento particular de la intimidad emocional, sin que haya redundancia con el otro factor. Además, los índices de confiabilidad obtenidos sugieren, según lo establece Kline (2000), que el instrumento cuenta con la suficiente consistencia interna para ser utilizado como instrumento de medición científica en investigaciones en Puerto 
Rico y América Latina, o como método de avalúo en procesos de psicoterapia y consejería.

En términos de las implicaciones teóricas del estudio, los hallazgos psicométricos demostraron que el instrumento replica las dos dimensiones consideradas por el autor en la construcción teórica del mismo y en la redacción de los ítems: cercanía emocional y distancia emocional. La cercanía emocional se refiere a aquellos sentimientos dentro de una relación que promueven el acercamiento, el vínculo, la conexión y, principalmente, la autorrevelación. Específicamente, este factor evalúa la escucha atenta (ítem 1), empatía (ítem 2) y comprensión por parte de la pareja (ítem 3), confianza (ítem 4) y autorrevelación (ítem 7). Por otra parte, la distancia emocional se refiere a aquellos sentimientos dentro de una relación que promueven el alejamiento, la desconexión y la necesidad de mayor afecto por parte de la pareja. En la escala, este factor examina el descuido emocional (ítem 5), soledad (ítem 6), distanciamiento emocional (ítem 8), necesidad de afecto (ítem 9) y desconexión emocional (ítem 10). A su vez, los resultados del análisis de correlación (validez convergente) entre la escala de intimidad emocional y la escala de calidad de la relación de pareja son consistentes con la literatura académica que evidencia una asociación fuerte entre la intimidad emocional y la satisfacción en la relación de pareja (Dandeneau \& Johnson, 1994; Dandurand \& Lafontaine, 2013; Greeff \& Malherbe, 2001).

En cuanto a las implicaciones prácticas del estudio, se demostró que el instrumento puede ser utilizado para el desarrollo de nuevas investigaciones en el campo de la psicología de las relaciones de pareja. El lenguaje sencillo y la estructura breve del mismo permiten que sea de fácil administración, evaluación e interpretación. En este sentido, este instrumento se constituye como una herramienta práctica y efectiva en el campo de la psicoterapia de parejas. Sus puntuaciones pueden ser calculadas de dos formas: mediante la sumatoria de sus 10 ítems, para obtener un índice general de intimidad emocional, o mediante la sumatoria de los cinco ítems de cada factor por separado. Los ítems pertenecientes al factor distancia emocional se puntúan de forma inversa.

Al igual que toda investigación, nuestro estudio no está exento de limitaciones. Primero, la muestra fue recopilada de forma accidental y no fue aleatoria. Segundo, no se pudo establecer la confiablidad del instrumento a través del tiempo, solo se pudo hacer a través de sus elementos. Por último, el procedimiento para recoger los datos no fue estándar, esto puede afectar las medias del estudio y aumentar el error estándar de medición.

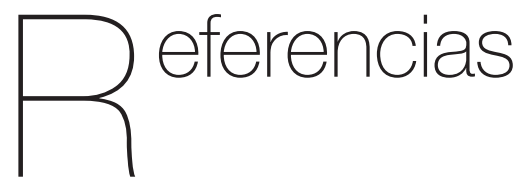

Ato, M., López, J. J., \& Benavente, A. (2013). Un sistema de clasificación de los diseños de investigación en psicología. Anales de Psicología, 29(3), 1038-1059.

Beckerman, N. (2002). Couples coping with discordant HIV status. AIDS Patient Care STDS, 16, 55-59. 
Byrne, B. M. (2010). Structural equation modeling with AMOS: basic concepts, applications, and programming (multivariate applications series). New York: Taylor \& Francis Group.

Dandeneau, M. \& Johnson, S.M. (1994). Facilitating intimacy: Interventions and effects Journal of Marital and Family Therapy, 28, 17-33

Dandurand, C. \& Lafontaine, M.F. (2013). Intimacy and Couple Satisfaction: The Moderating Role of Romantic Attachment. International Journal of Psychological Studies, 5(1), 74-90. doi:10.5539/ijps. v5n1p74

DeRogatis, L.R. (2008). Assessment of sexual function/dysfunction via patient reported outcomes. International Journal of Impotence Research, 20(1), 35-44. doi:10.1038/sj.ijir.3901591

Greeff, A. P., \& Malherbe, H. L. (2001). Intimacy and marital satisfaction in spouses. Journal of Sex \& Marital Therapy, 27(3), 247-257. doi:10.1080/009262301750257100

Hair, J. F., Black, W. C., Babin, B. J., Anderson, R. E., \& Tatham, R. L. (2009). Análise multivariada de dados. Porto Alegre: Bookman Editora.

Hand, M.M., Thomas, D., Buboltz, W.C., Deemer, E.D., \& Buyanjargal, M. (2013). Cyberpsychology, Facebook and Romantic Relationships: Intimacy and Couple Satisfaction Associated with Online Social Network Use. Behavior and Social Networking, 16(1), 8-13. doi:10.1089/ cyber.2012.0038

Hatcher, L. (1994). A step-by-step approach to using the SAS system for factor analysis and structural equation modeling. Cary, NC: SAS Institute Inc.

Hendrick, S. S. (1988). A generic measure of relationship satisfaction. Journal of Marriage and the Family, 50(1), 93-98.

Hetherington, S. E., \& Soeken, K. L. (1990). Measuring changes in intimacy and sexuality: a self-administered scale. Journal of Sex Education and Therapy, 16(3), 155-163.

Hu, L. T., \& Bentler, P. M. (1999). Cutoff criteria for fit indexes in covariance structure analysis: Conventional criteria versus new alternatives. Structural Equation Modeling: a Multidisciplinary Journal, 6(1), 1-55.

loseba, M.S., \& Martinez-Pampliega, A. (2009). Adaptación y estudio psicométrico de dos instrumentos de pareja: Índice de Satisfacción Matrimonial y Escala de Inestabilidad Matrimonial. Revista de Investigación en Psicología, 12(2), 177-192. doi:10.15381/rinvp.v12i2.3763

Kline, P. (2000). The Handbookofpsychometric testing. New York: Routledge.

Laurenceau, J. P., Barrett, L. F., \& Pietromonaco, P. R. (1998). Intimacy as an interpersonal process: The importance of self-disclosure, partner disclosure, and perceived partner responsiveness in interpersonal exchanges. Journal of Personality and Social Psychology, 74(5), 1238-1251.

Maureira, F. (2016). Construcción y validación de una escala para medir los cuatro componentes del amor de pareja. Gaceta de Psiquiatría Universitaria, 12(1), 99-105. 
Mazadiego, T.J., \& Garcés, J.R.N. (2011). amor medido por la escala triangular de Sternberg. Psicolatina, 22, 1-10.

Montero, I., \& León, O. G. (2007). A guide for naming research studies in Psychology. International Journal of Clinical and Health Psychology, 7(3), 847-862.

Pierce, G. R. (1994). The Quality of Relationships Inventory: Assessing the interpersonal context of social support. In B. R. Burleson, T. L. Albrecht, \& I. G. Sarason (Eds.), Communication of social support: Messages, interactions, relationships, and community (pp. 247264). Sage Publications, Inc.

Satorra, A., \& Bentler, P. M. (2001). A scaled difference chi-square test statistic for moment structure analysis. Psychometrika, 66(4), 507-514.

Schaefer, M. T., \& Olson, D. H. (1981). Assessing intimacy: The PAIR inventory. Journal of Marital and Family Therapy, 7(1), 47-60.
Sternberg, R. J. (1986). A triangular theory of love. Psychological Review, 93(2), 119-135.

Sternberg, R. (1988). Teoría Triangular del Amor. Madrid: editorial Paidós

Vohs, K. D., \& Baumeister, R. F. (2004). Understanding self-regulation: An introduction. Handbook of self-regulation: Research, theory, and applications, 1-9.

Waring, E. M., \& Reddon, J. R. (1983). The measurement of intimacy in marriage: The Waring Intimacy Questionnaire. Journal of Clinical Psychology, 39(1), 53-57.

Zimmer-Gembeck, M.J., Arnhold, V. \& Connolly, J. (2014). Intercorrelations of Intimacy and Identity Dating Goals with Relationship Behaviors and Satisfaction among Young Heterosexual Couples. Social Sciences, 3(1), 44-59. 